\title{
Wind Turbine Fault Detection Using Counter-Based Residual Thresholding *
}

\author{
Ahmet Arda Ozdemir, Peter Seiler, and Gary J. Balas* \\ * Department of Aerospace Engineering and Mechanics, \\ University of Minnesota, Minneapolis, MN 55455, USA \\ (e-mail: arda@aem.umn.edu, seiler@aem.umn.edu, \\ balas@aem.umn.edu)
}

\begin{abstract}
This paper investigates application of model-based fault detection techniques on wind turbines. Fault residuals are generated through physically redundant sensors, parity equations and common filtering methods. Up-down counters are used for decisioning on these fault residuals. These simple counters are commonly used in the aerospace industry to improve missed detection rates. These techniques constitute an easily implementable fault detection and isolation system on an industrial turbine. The performance of the developed algorithm is evaluated on a model of a commercial sized 4.8MW wind turbine. Realistic fault scenarios in the sensing, actuation and drivetrain subsystems are considered. It is seen that most faults can be detected with fast detection times and minimal false alarms without implementation of more complex filtering and detection techniques on residuals.
\end{abstract}

Keywords: Fault detection, Counters, Threshold selection, Wind Turbine

\section{INTRODUCTION}

Wind energy is a rapidly growing renewable energy source as its cost per unit is reaching competitive levels. Maintenance and repair costs constitute an important portion of the operating costs of a typical wind turbine. These costs are more significant for offshore wind turbines which are located further from the maintenance centers. In addition, offshore wind turbines in general have lower availability rates than their onshore counterparts due to the longer repair times.

Fault detection and isolation (FDI) algorithms can be used to detect the irregularities in the sensing and actuation subsystems of the wind turbine and accommodate these faults when possible. In addition, FDI systems can also be used for detection of the precursors of some critical failures, which may otherwise result in a high cost breakdown in the future. These benefits of the FDI systems can significantly reduce the downtime and repair costs of the wind turbine over its lifetime.

The fault detection problem usually comprises a method to compute residuals and a process to declare faults based on the residuals. It is desired that the generated residual be a good representation of the fault of interest while being insensitive to process and measurement noises. Generation of residuals depends on the information available about

\footnotetext{
* This work was supported by the University of Minnesota Institute on the Environment, IREE Grant No. RS-0039-09, the US Department of Energy Contract No. DE-EE0002980 and the US National Science Foundation under Grant No. NSF-CNS-0931931. Any opinions, findings, and conclusions or recommendations expressed in this material are those of the author(s) and do not necessarily reflect the views of the University of Minnesota, Department of Energy or National Science Foundation.
}

the system. If a sufficiently accurate model of the system is available, model based methods can be used to estimate system states and outputs. Donders (2002) applied Kalman Filter and Interacting Multiple-Model estimators to the wind turbine FDI problem. $H_{\infty} / H_{-}$techniques for observer design were used by Wei and Verhaegen (2008) and Szaszi et al. (2002). Another method of obtaining residuals is to compare the redundant information about the system if the system has some physical redundancy built in it. See Gertler (1998), Isermann (2005), and Ding (2008) for a detailed treatment of model based and modelfree fault detection methods.

A common method used for decision making in fault detection algorithms is thresholding. That is, a fault is declared if the residual exceeds a certain threshold. See Gertler (1998), Emami-Naeini et al. (1988) for applications of fixed thresholding and Stoustrup et al. (2003) for time varying thresholding. Another method used for decisioning is up-down counters. These are also known as leaky bucket counters in the communications and software literature. See Logothetis and Trivedi (1994) and Butto et al. (1991) for the applications of leaky bucket counters in the communications literature. These counters are also commonly used in aerospace industry for fault detection purposes. Gertler (1998) used a special case of the up-down counters for fault detection where the up and down-counts at each time step are simply set equal to one. The operation of these counters is explained in detail in Section 4 .

This paper develops a fault detection and isolation system for a typical commercial wind turbine. Faults are considered in the pitch actuators and sensors, rotor speed and generator speed sensors as well as system faults in the drivetrain and generator. Both model based and physical 
redundancy based residuals are used. Up-down counters are used for decisioning on each residual.

The paper has the following structure: Section 2 describes the wind turbine model used in this paper and explains the faults that are considered. Section 3 explains the design of the FDI system in detail. The design and analysis of updown counters for FDI decisioning is covered in Section 4. The results are presented in Section 5. Conclusions are presented in Section 6.

\section{WIND TURBINE MODEL AND FAULT DESCRIPTIONS}

The wind turbine model and problem considered in this paper is based on the work presented in Odgaard et al. (2009). The modeled turbine is a variable speed, three bladed horizontal axis wind turbine with a rated power of 4.8 MW. A brief overview of the problem formulation is presented in this section.

\subsection{Aerodynamics and Pitch System Model}

The captured power is approximately given by:

$$
P_{r}=\tau_{\text {aero }} \omega_{r}=\frac{1}{2} \rho A v^{3} C_{p}(\lambda, \beta)
$$

where $\tau_{\text {aero }}(\mathrm{N})$ is the aerodynamic torque, $\omega_{r}(\mathrm{rad} / \mathrm{s})$ is the rotor speed, $\rho\left(\mathrm{kg} / \mathrm{m}^{3}\right)$ is the air density, $A\left(\mathrm{~m}^{2}\right)$ is the area swept by the rotor, $v(\mathrm{~m} / \mathrm{s})$ is the wind speed. $C_{p}$ (unitless) is the power coefficient which represents how much of the power available in wind is captured. $C_{p}$ is a function of blade pitch angle $\beta$ (deg) and tip speed ratio $\lambda$ (unitless) where $\lambda$ is defined as:

$$
\lambda=\frac{\omega_{r} R}{v}
$$

$R(\mathrm{~m})$ is the rotor radius. From Equation (1), it is seen that the aerodynamic torque can be written as:

$$
\tau_{\text {aero }}=\frac{\rho A v^{3} C_{p}(\lambda, \beta)}{2 \omega_{r}}
$$

The turbine pitch actuators are modeled by a second-order transfer function $G_{a c t}(s)$ :

$$
\frac{\beta_{j}(s)}{\beta_{j, r e f}(s)}=G_{a c t}(s)=\frac{\omega_{n}^{2}}{s^{2}+2 \zeta \omega_{n} s+\omega_{n}^{2}}
$$

where $j=1,2,3$ denotes the blade index. The nominal values of $\zeta$ and $\omega_{n}$ are 0.6 and $11.11 \mathrm{rad} / \mathrm{s}$ respectively.

\subsection{Drivetrain and Generator Model}

Drivetrain flexibility is modeled by a two mass model:

$$
\left[\begin{array}{c}
\dot{\omega}_{r} \\
\dot{\omega}_{g} \\
\dot{\theta}_{\Delta}
\end{array}\right]=A\left[\begin{array}{c}
\omega_{r} \\
\omega_{g} \\
\theta_{\Delta}
\end{array}\right]+\left[\begin{array}{cc}
-1 / J_{r} & 0 \\
0 & 1 / J_{g} \\
0 & 0
\end{array}\right]\left[\begin{array}{c}
\tau_{\text {aero }} \\
\tau_{g}
\end{array}\right]
$$

where $\omega_{r}$ is the rotor speed, $\omega_{g}$ is the generator speed, $\theta_{\Delta}$ is the drivetrain torsion. The state matrix $\mathrm{A}$ is given by:

$$
A=\left[\begin{array}{ccc}
\frac{-\left(B_{d t}+B_{r}\right)}{J_{r}} & \frac{B_{d t}}{N_{g} J_{r}} & \frac{-K_{d t}}{J_{r}} \\
\frac{\eta_{d t} B_{d t}}{N_{g} J_{g}} & \frac{-\eta_{d t} B_{d t}-B_{g} N_{g}^{2}}{N_{g}^{2} J_{g}} & \frac{\eta_{d t} K_{d t}}{N_{g} J_{g}} \\
1 & -\frac{1}{N_{g}} & 0
\end{array}\right]
$$

$J_{r}$ and $J_{g}$ are the rotor and generator inertia. $B_{r}$ and $B_{g}$ are the viscous damping of the rotor and generator. $B_{d t}$ and $K_{d t}$ are the damping and stiffness coefficients of the drivetrain flexibility, and $N_{g}$ is the gearbox ratio.

The generator dynamics are modeled with a first-order transfer function:

$$
\frac{\tau_{g}(s)}{\tau_{g, \text { ref }}(s)}=G_{g e n}(s)=\frac{\alpha_{g}}{s+\alpha_{g}}
$$

The nominal value of $\alpha_{g}=50$. Numeric values of the constant given in this section is summarized in Table 1.

Table 1. List of Turbine Parameters

\begin{tabular}{ccc} 
Symbol & Value & Description \\
\hline$R$ & $57.5 \mathrm{~m}$ & Rotor radius \\
$B_{r}$ & $7.11 \mathrm{~kg} / \mathrm{s}$ & Rotor viscous damping \\
$B_{g}$ & $45.6 \mathrm{~kg} / \mathrm{s}$ & Generator damping \\
$J_{r}$ & $55 \times 10^{6} \mathrm{~kg} \mathrm{~m} \mathrm{~m}^{2}$ & Rotor inertia \\
$J_{g}$ & $390 \mathrm{~kg} \mathrm{~m}$ & Generator inertia \\
$B_{d t}$ & $775.49 \mathrm{~kg} / \mathrm{s}$ & Drivetrain damping \\
$K_{d t}$ & $2.7 \times 10^{9} \mathrm{~kg} / \mathrm{s}^{2}$ & Drivetrain stiffness \\
$\eta_{d t}$ & 0.97 & Drivetrain efficiency \\
$N_{g}$ & 95 & Gearbox ratio
\end{tabular}

\subsection{Turbine Control System}

Wind turbine operation is separated into four regions depending on wind speed. These regions are defined by cut-in, rated and cut-out wind speeds. The wind turbine is not operated if the wind speed is below cut-in wind speed. This region is called Region 1 . The turbine is in Region 2 when the wind speed is between cut-in and rated wind speeds. The turbine control system aims to maximize power capture in this region. The turbine can capture its rated power when the wind speed is above its rated wind speed (Region 3). This maximum power is limited by the turbine generator. The control objective in this region is to main the rated power while reducing structural loads. Turbines are shut down to avoid structural damage if the wind speed exceeds cut-out wind speed (Region 4). This turbine model focuses on Region 2 and 3 operation. The control system of the turbine includes two separate controllers for Region 2 and Region 3 operation. Its cut-in, rated and cut-out wind speeds are given as $0 \mathrm{~m} / \mathrm{s}, 12.5 \mathrm{~m} / \mathrm{s}$ and $25 \mathrm{~m} / \mathrm{s}$ respectively.

In Region 2 the performance objective is to maximize the captured power. The power coefficient $C p$ has a maximum at an optimal blade pitch $\beta_{\text {opt }}$ and tip speed ratio $\lambda_{\text {opt }}$. The captured power is maximized by controlling the turbine such that it operates at this peak operating condition. A common control Region 2 control law is:

$$
\begin{gathered}
\tau_{g, c m d}=\frac{1}{2} \rho A R^{3} \frac{C_{p_{\max }}}{\lambda_{o p t}^{3}} \omega_{r}^{2} \\
\beta_{j, c m d}=\beta_{o p t}
\end{gathered}
$$

This control strategy holds all blades fixed at the pitch required for optimal power capture. The generator torque command is proportional to the square of the rotor speed. In steady wind conditions this control law drives the rotor speed to the value required to achieve the optimal tip speed ratio. Further details on this control can be found in Johnson et al. (2006). 
In Region 3 the performance objective is to maintain the rated power while reducing structural loads. One means of achieving this goal is to use collective blade pitch control to shed the extra power in the wind and minimize variations in the rotor speed. The generator torque is used to obtain the rated power. Minimizing rotor speed variations is important to avoid over-speeding of the generator. It is also correlated with exciting the structural loads of the turbine. A discrete PI controller with $K_{i}=1, K_{p}=4$ and sampling time $T_{s}=0.01 s$ is used for collective blade pitch control. The generator torque is set as:

$$
\tau_{g, \text { ref }}=\frac{P_{\text {rated }}}{\omega_{g}}
$$

This simple control scheme does not include more advanced schemes such as individual pitch control. The FDI system presented in this paper is not specific to this control scheme and can be implemented on any control law without any modifications.

\subsection{Sensor Configuration}

Typically, wind turbines are built with sensor configurations that involve some physical redundancy for fault tolerant operation. The available sensors on the model are listed in Table 2. Subscripts _ $m 1$ and _ $m 2$ represent redundant measurements of the quantities. In addition to the measurements listed in Table 2, it is assumed that the digital controller commands are also available for the FDI system. The sampling time of the sensors is assumed to be $T_{s}=0.01$ seconds.

Table 2. Sensor Configuration of Wind Turbine

\begin{tabular}{lll} 
Sensor & Symbols & Noise $\left(\mu, \sigma^{2}\right)$ \\
\hline Rotor Speed & $\omega_{r_{-} m 1}, \omega_{r_{-} m 2}(\mathrm{rad} / \mathrm{s})$ & $(0,0.025)$ \\
Generator Speed & $\omega_{g_{-} m 1}, \omega_{g_{-} m 2}(\mathrm{rad} / \mathrm{s})$ & $(0,0.050)$ \\
Generator Torque & $\tau_{g_{-} m}(\mathrm{Nm})$ & $(0,90)$ \\
Generator Power & $P_{g_{-} m}(\mathrm{~W})$ & $(0,1000)$ \\
i-th Blade Pitch Angle & $\beta_{i_{-} m 1}, \beta_{i_{-} m 2}(\mathrm{deg})$ & $(0,0.200)$ \\
Wind Speed & $v_{m}(\mathrm{~m} / \mathrm{s})$ & $(1.5,0.500)$
\end{tabular}

The noises acting on each sensor are assumed to be Gaussian white noise. The mean values $(\mu)$ and variances $\left(\sigma^{2}\right)$ of the sensor noises are given in Table 2. The wind speed sensor is considered to have low accuracy and it requires frequent calibration. In addition, note that the standard deviation of the rotor speed measurement is $\sigma \approx 0.16 \mathrm{rad} / \mathrm{s}$. This corresponds to approximately $10 \%$ of the rated rotor speed of $1.7 \mathrm{rad} / \mathrm{s}$. The large noise on this measurement was an issue in our baseline designs.

\subsection{Fault Modeling and Detection Requirements}

The sensor faults considered in this paper are listed in Table 3. It is required that these faults (Faults 1-5) are detected in $10 T_{s}$, i.e. 10 sampling periods of the sensors.

Actuator and system level faults are listed in Table 4. Fault 6 is a hydraulic failure in pitch actuator 2 which involves an abrupt change in pitch actuator model parameters $\zeta$ and $\omega_{n}$. The second scenario, Fault 7, involves increased air content in the actuator oil which manifests itself with slower actuator response over time. This fault is modeled as a slow, time varying change in actuator parameters $\zeta$ and $\omega_{n}$. Bode plots of the nominal and faulty actuator
Table 3. List of Sensor Faults

\begin{tabular}{cll} 
Fault No & Fault & Description \\
\hline 1 & $\beta_{1 \_m 1}=5 \mathrm{deg}$ & Pitch Sensor Stuck \\
2 & $\beta_{2 \_m 2}=1.2 \beta_{2 \_m 2}$ & Pitch Sensor Scale Factor \\
3 & $\beta_{3 \_m 1}=10 \mathrm{deg}$ & Pitch Sensor Stuck \\
4 & $\omega_{r \_m 1}=1.4 \mathrm{rad} / \mathrm{s}$ & Rotor Speed Sensor Stuck \\
5 & $\omega_{r \_m 2}=1.1 \omega_{r \_m 2}$ & Simultaneous Rotor Speed and \\
& $\omega_{g \_m 2}=0.9 \omega_{g \_} m 2$ & Gen. Speed Sensor Scale Factor
\end{tabular}

models are shown in Figure 1. Note that the air-in-fault enters the system gradually and Figure 1 only shows the worst case operation. Detection time requirements for Fault 6 and 7 are given as $8 T_{s}$ and $600 T_{s}$ respectively.
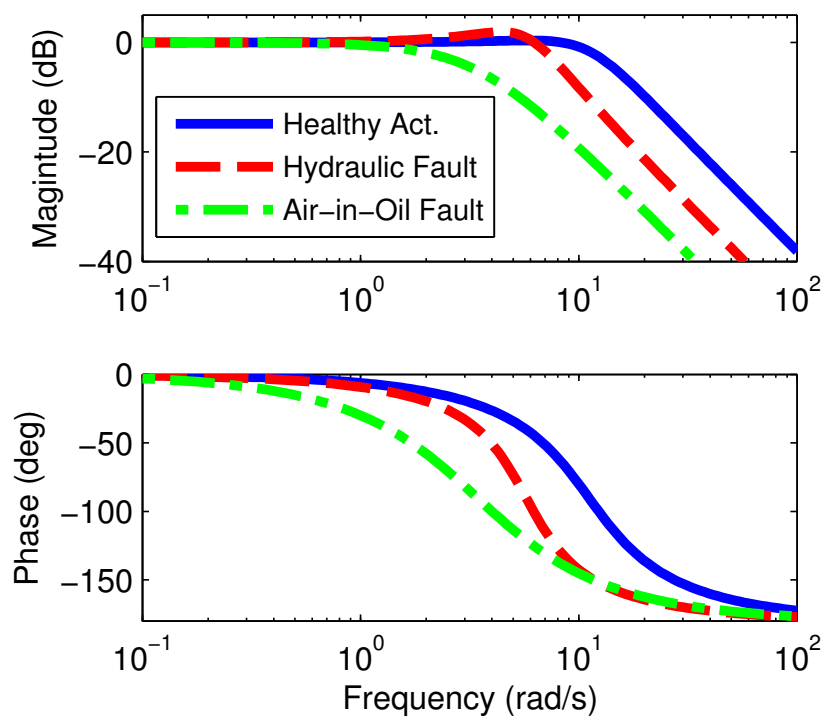

Fig. 1. Bode Plots for Healthy and Faulty Actuator Models

In addition, two system level faults are considered (Faults 8 and 9). The first system level fault, Fault 8, involves an offset in the inner control loop of the generator. It results in a bias in the generator torque $\tau_{g}=\tau_{g}+100(\mathrm{Nm})$. The second system level fault considered is increased friction in the drivetrain (Fault 9). This fault typically develops slowly over time and results in increased vibrations in the drivetrain. This fault is modeled with a $5 \%$ change in the drivetrain efficiency $\eta_{d t}$. Fault 8 needs to be detected in $5 T_{s}$ and there are no time restrictions for the detection of Fault 9. A list of the actuator and system level faults are given in Table 4.

Table 4. List of Actuator and System Faults

\begin{tabular}{cl} 
Fault No & Description \\
\hline 6 & Pitch Actuator 2 Hydraulic Failure \\
7 & Pitch Actuator 3 Air in Oil Failure \\
8 & Generator Inner Control Loop Failure \\
9 & Increased Drivetrain Friction due to Wear
\end{tabular}

In addition to the detection time constraints, it is required that the false alarm rate be less than 1 in $10^{5}$ time steps. It is also expected that the false alarms are cleared in three time steps. 


\section{FAULT DETECTION AND ISOLATION}

\subsection{System Overview}

The proposed method for detecting turbine faults relies on the standard residual generation and decision logic architecture shown in Figure 2. Sensor measurements, blade pitch commands and generator torque commands are used to generate various fault residuals. These residuals are selected to be representative of turbine faults of interest. Details of the residual generation are presented in Section 3.2. Individual up-down counters are used as a thresholding logic for each residual. These counters output a flag indicating if the values of these residuals are within normal operation limits. The up/down counters are, compared to the other algorithm aspects, relatively non-standard. Thus these are described in more detail in Section 4. Finally, the fault isolation system decides the faulty component on the turbine based on the flags from the up-down counters. Details of the fault isolation logic is presented in 3.3.

Fig. 2. FDI System Overview

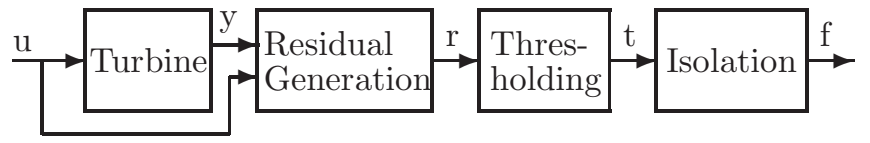

\subsection{Residual Generation Methods}

The residual generation system takes sensor measurements and controller commands and outputs a vector of residuals. This output vector is denoted as $r . i^{t h}$ element of this vector is denoted as $r(i)$. A summary of each fault and the corresponding residuals are presented in Table 5. The remainder of this section describes the various methods used for generating these residual signals.

Table 5. Summary of Residual Residuals

\begin{tabular}{ll} 
Fault No & Residual Types \\
\hline $1,2,3$ & Physical Red., Parity Eq. \\
4,5 & Physical Red. \\
6,7 & Physical Red., Parity Eq. \\
8 & Parity Equations \\
9 & Data Driven Methods
\end{tabular}

Physical Redundancy A residual $r$ can be generated by direct comparison of the physically redundant measurements of same quantity $m_{1}$ and $m_{2}$ :

$$
r=m_{1}-m_{2}
$$

If the system is healthy then $r$ is only a function of the sensor noise properties. If one of the sensors is faulty then the residual is a function of the fault and the noise properties. The disadvantage of this approach is that the residual typically carries a larger variance than the individual noises. Specifically, the variance of $r$ in the fault free case is given by:

$$
\operatorname{Var}[r]=\operatorname{Var}\left[m_{1}\right]+\operatorname{Var}\left[m_{2}\right]+2 \operatorname{Cov}\left[m_{1}, m_{2}\right]
$$

Generally it is assumed that the measurement noises from redundant sensors are independent and identically distributed. For this case, the variance of the residual is twice as large as the sensor noise variance, i.e.

$$
\operatorname{Var}[r]=2 \operatorname{Var}\left[m_{1}\right]=2 \operatorname{Var}\left[m_{2}\right]
$$

Residual generation via physically redundant measurements is used to detect faults 1-7. Residual generation for each of these faults described in more detail below.

Each blade has two pitch angle sensors and these redundant measurements can be used for detecting faults $1,2,3,5$ and 6 . Two physically redundant pitch angle measurements are subtracted from each other to generate one residual for each blade:

$$
r(j)=\beta_{j_{-} m 1}-\beta_{j_{-} m 2}
$$

where $\mathrm{j}=1,2,3$ is the blade index.

The turbine system contains two rotor speed and two generator speed measurements. The gearbox system has a high stiffness and its dynamics are significantly faster than the rotor and generator dynamics. Hence the rotor speed and generator speed can be approximately related as $\omega_{r}=\omega_{g} / N_{g}$ where $N_{g}$ is the drivetrain ratio. Relating two rotor speed measurements with two generator speed measurements yields a total 4 rotor speed measurements. The worst case scenario considered for these sensors involves a simultaneous failure of two sensors (Fault 5). A fifth measurement is required for isolation in this two failure case. This fifth rotor speed measurement is obtained synthetically via generator power and torque measurements as $\omega_{r}=P_{g_{m}} /\left(\tau_{g_{m}} N_{g}\right)$. A median value filter is used to pick a healthy measurement from the five redundant measurements. The median filter is equal to one of the three healthy measurements even when two of the five sensors are affected by a large fault. Let $\omega_{r_{\text {_median }}}$ be the healthy measurement from the median value filter. $\omega_{r_{-} \text {median }}$ is obtained as:

$\omega_{r \_ \text {median }}=$ median $\left(\omega_{r_{\_} m 1}, \omega_{r_{-} m 2}, \frac{\omega_{g \_m 1}}{N_{g}}, \frac{\omega_{g_{-} m 2}}{N_{g}}, \frac{P_{g_{m}}}{\tau_{g_{m}} N_{g}}\right)$

Five residuals $r(4,5,6,7,8)$ are obtained by comparing $\omega_{r_{-} \text {median }}$ to each one of the five measurements. The rotor speed measurements have a low signal-to-noise ratio. Hence the rotor speed rotor speed residuals are averaged over 40 time steps to reduce the variance of the Gaussian white noise in the steady state. This corresponds to the digital filter $F_{\omega_{r}}(z)$ :

$$
F_{\omega_{r}}(z)=\frac{1}{40} \frac{z^{40}+z^{39}+\ldots+z^{2}+z^{1}+1}{z^{40}}
$$

These filtered residuals are used for the detection of faults 4 and 5 .

Parity Equations Model-based residuals can be obtained if a reasonably accurate model of the system and its inputs are available. The residual is generated by computing the system output with the ideal system response from the model and measured system inputs. An ideal blade pitch response signal is generated via the nominal actuator model and the pitch commands from the controller:

$$
\beta_{j *}(s)=G_{a c t}(s) \beta_{j, r e f}(s)
$$

where $\mathrm{j}=1,2,3$ is the blade index and * denotes the ideal response. Deviations from this ideal response can be used to detect faults in the pitch systems. Two residuals for each blade are obtained by comparing the ideal response to each 
of the two blade pitch measurements: $r(8+j)=\beta_{j *}-\beta_{j_{-} m 1}$ and $r(11+j)=\beta_{j *}-\beta_{j_{-} m 2}$ where $j=1,2,3$ is the blade index. Due to high noise levels, these six residuals have been filtered with a sixth-order elliptical filter. The elliptical filter was designed to have $1 \mathrm{~dB}$ pass-band ripple and $40 \mathrm{~dB}$ of attenuation at $100 \mathrm{rad} / \mathrm{s}$. The elliptical filter has DC gain of $-1 d B$. This non-unity DC gain does not hinder FDI performance because actuator faults are mainly observed in the mid-frequency range.

An ideal generator torque response is obtained using generator torque commands and a model for the generator system:

$$
\tau_{g *}(s)=G_{g e n}(s) \tau_{g, r e f}(s)
$$

A parity equation is obtained by comparing this ideal response to the generator torque measurement: $r(15)=$ $\tau_{g_{-} m}(t)-\tau_{g *}(t)$. This residual is used for the detection of the generator system fault (Fault 8).

Data Driven Methods The drivetrain system fault (Fault 9) constitutes a challenge for the model based FDI methods. This is due to two reasons. First, the $5 \%$ variation in the drivetrain efficiency $\eta_{d t}$ introduced by this fault has a minimal impact on the drivetrain model given in Eqs. (5) and (6). Second, the aerodynamic torque input, $\tau_{\text {aero }}$, to this model depends on unreliable wind speed measurements. Wind speed sensors have large measurement errors and require frequent calibration. In particular, the large wind speed measurement bias in this turbine model prohibits sufficiently accurate estimation of $\tau_{\text {aero }}$ from the power curve model (Eq. (3)).

An alternative approach is taken in this paper. This approach relies on using the correlations between blade pitch commands and wind speed measurements. The approach attempts to minimize the impact of errors in the wind speed measurement.

A residual for Fault 9 is generated via the correlation between blade pitch commands and wind speed measurements. When operating in Region 3, the turbine control system actively increases blade pitch angles to shed the extra power in the wind. When Fault 9 occurs the $5 \%$ extra power loss in the gearbox results in smaller pitch angle commands at a given wind speed. Figure 3 shows the distribution of the blade pitch angles versus wind speed measurements. A second order polynomial that is a function of wind speed is fitted to the healthy data points. Only data points with wind speeds higher than $14 \mathrm{~m} / \mathrm{s}$ and pitch angles higher than 2 deg are used for this fitting to ensure the wind turbine is operating in Region 3. The polynomial fit is given by:

$$
\hat{\beta}_{c m d}=0.0020 v_{m}^{2}+1.2928 v_{m}-15.2949
$$

The constant of this polynomial is reduced by -0.1 to ensure that on average the estimates are lower than the actual commands. This reduces sensitivity to false alarms since a larger fault is required to push the actual pitch commands lower than the estimated one. A residual is then defined as:

$$
r(16)=\beta_{c m d}-\left(0.0020 v_{m}^{2}+1.2928 v_{m}-15.2949\right)
$$

Thresholding algorithm processes this residual as it is without taking its absolute value.
This approach requires gathering of wind speed measurements and controller pitch commands for some duration assuming healthy operation. Then the data-driven correlation model has all the sensor bias, scale factor errors etc. built into the model. However, one challenge with this approach is that the frequent calibration of the wind speed sensors would require a new fit each time. The presented method is a very basic data driven approach. More sophisticated data driven approaches can be used tackle these issues.

\section{Fig. 3. Pitch Angles versus Wind Speed Distribution}

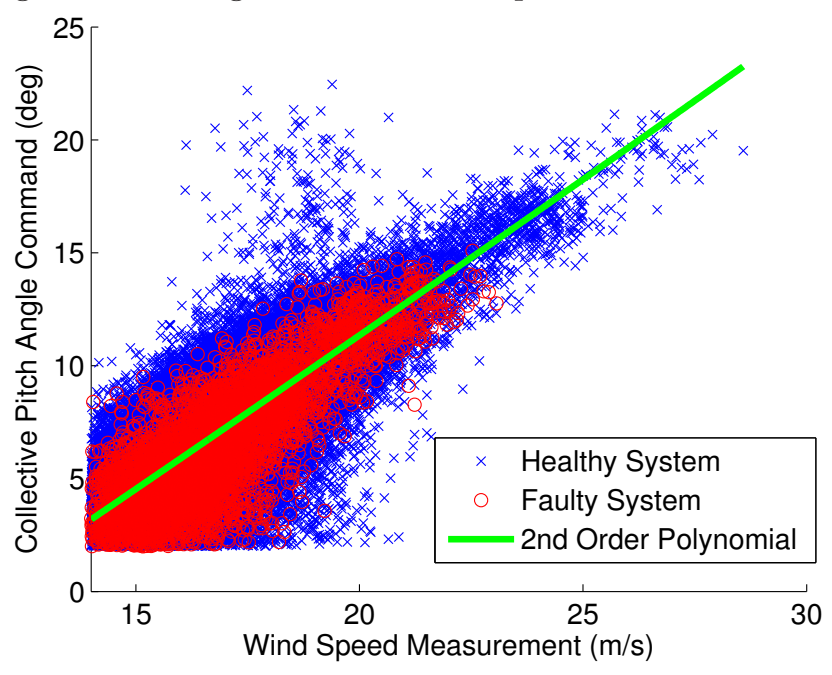

\subsection{Fault Isolation}

The residuals described in the previous section are passed through up-down counters for decision logic. The up-down counters, described further in Section 4, output binary healthy or faulty operation flags for each residual. The fault isolation system takes these flags and attempts to isolate the faulty turbine component.

Faults in the pitch sensor and actuator systems can be isolated using three residuals generated for each blade. The faults are isolated using a basic fault symptom table approach (Isermann (2005)). The basic isolation approach is described for blade 2 sensor and actuator failures. Three residuals are generated for blade 2. $r(2)=\beta_{2 \_m 1}-\beta_{2 \_m 2}$ is the difference between the sensor pitch angle measurements for blade 2. $r(9)=\beta_{2 *}-\beta_{2 \_m 1}$ and $r(12)=\beta_{2 *}-$ $\beta_{2 \_m 2}$ are the difference between the ideal pitch response and each sensor measurement. The fault symptom matrix for these three residuals is shown in Table 6. In this table "0" denotes a healthy flag from the up-down counter and "1" denotes a fault flag from the up-down counter. For example, the symptoms of a failure in the 2nd pitch actuator (Fault 6) will appear in residuals 9 and 12. In particular, both sensor measurements will deviate from the ideal, modeled output. However, the comparison of the blade pitch sensors, residual 2, will indicate healthy operation in the presence of fault 6 . On the other hand, a failure of the second pitch actuator measurement will appear in residuals 2 and 12 . These two residuals indicate that the second measurement differs from both the first measurement and the ideal actuator model. Residual 9 compares the first measurement to the ideal response and 
will continue to indicate healthy operation in the presence of a fault to sensor 2. The faults to the blade pitch angles and blade pitch actuator (Faults 1,2,3,6, and 7) are all isolatable from the generated residual using this simple fault symptom table approach.

Table 6. $2^{\text {nd }}$ Blade Pitch System Fault Symptom Table

\begin{tabular}{ccccc} 
Fault No & $\mathrm{r}(2)$ & $\mathrm{r}(9)$ & $\mathrm{r}(12)$ & Decision \\
\hline- & 1 & 1 & 0 & Sensor $1\left(\beta_{2 \_} \_\right.$1 $)$Faulty \\
2 & 1 & 0 & 1 & Sensor $2\left(\beta_{2 \_} 2\right)$ Faulty \\
6 & 0 & 1 & 1 & $2^{\text {nd }}$ Pitch Actuator Faulty
\end{tabular}

Faults 4 and 5 represent failures in the two rotor and generator speed sensors. Residuals $r(4,5,6,7,8)$ are used for detection of these faults. Residual 4 compares rotor speed sensor $1\left(\omega_{r \_m 1}\right)$ with the output of the median filter. If the up-down counter flags this residual then this indicates a failure in sensor 1 . No isolation is necessary. Similarly flags on residuals 5, 6, 7 directly indicate failures in the remaining rotor and generator speed sensors $\left(\omega_{r_{-} m 2}\right.$, $\left.\omega_{g_{-} m 1}, \omega_{g_{-} m 2}\right)$. These residuals are not affected by the faults in the other components of the system. Hence the decision flag from up-down counters are directly sufficient for declaring a fault. Residual 8 involves the synthetic rotor speed measurement from generator power and torque measurements. A flag on this residual indicates a failure in either the power or generator torque measurements.

Fault 8 represents a failure in the generator system. Residual 15 represents the error between the ideal generator torque response from generator model and the measurement. This residual is not affected by other faults in the system. Hence no specific fault isolation is required. A fault is declared on the flag from the up-down counters.

An efficiency loss in the drivetrain system (Fault 9) is monitored through residual 16. This residual represents the difference between actual and estimated blade pitch commands. These estimated blade pitch commands are based on wind speed measurements. Turbine control system generates blade pitch commands based on the generator speed measurements. Hence a fault in the generator speed sensors can cause a false flag on this residual. Therefore this fault is declared only when at least one of the generator speed measurements is healthy.

\section{UP-DOWN COUNTERS}

This section presents the use of up-down counters as a thresholding algorithm. The up-down counter involves discrete dynamics that make use of the allowed detection time limits. The aim is to increase the fault detection probability while keeping the false alarm rate constant. If desired, a better false alarm rate can be obtained by sacrificing from this improved detection rate. Section 4.1 describes the operation of the up-down counters. Section 4.2 describes analysis of these counters. This analysis is based on the assumption that the residual is the sum of a fault signal and uncorrelated, zero-mean Gaussian noise. This analysis is particularly useful for residuals generated based on physical redundancy since there are no dynamics introduced by fault detection filters.

\subsection{Structure of the Up-Down Counters}

The structure of the discrete-time up-down counter is shown in Figure 4. At each time step an up-count is triggered if the residual exceeds $T_{o l}$ otherwise the counter counts down by $-D$. A fault is declared if the value of the counter exceeds $\mathrm{Tol}_{2}$. Discrete time up-down counters involve six parameters in the most general case: upcount amount $+U$, down-count amount $-D$, up-counting threshold $T o l_{1}$, fault declaration threshold $T_{0} l_{2}$, counter lower-bound $L B$, and counter upper-bound $U B$. The upcount frequency is adjusted by the $T o l_{1}$ threshold. Upcount and down-count parameters $+U$ and $-D$ introduce a penalty on the residual exceeding the $T o l_{1}$ threshold.

\section{Fig. 4. Up/Down Counter Structure}

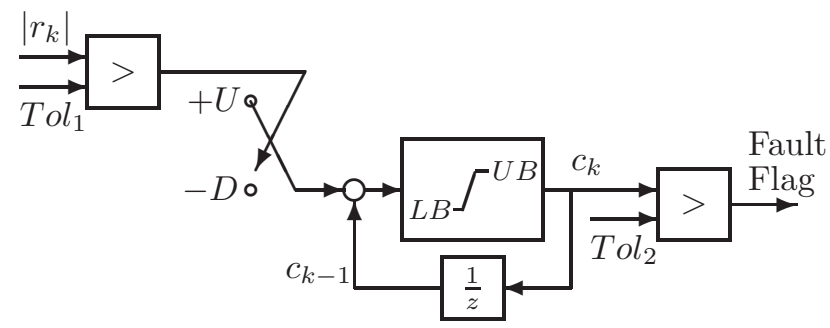

Constant (time-invariant) counter parameters are used in this work. All parameters except Tol $_{1}$ are chosen as integers for ease of design and implementation. The parameters $+U,-D, T_{2}, L B$, and $U B$ can be scaled together without any effect in general. $-D=-1$ is chosen to eliminate one parameter from the counter design. $+U$ is chosen as an integer. This scaling may not be possible when the parameters of the counters are integers. However, the error introduced by this approximation is typically small since the discarded fractions are small compared to the upper-bound of the counter. As a starting point, $L B=0$ and $U B=255$ are chosen so that the counter can be implemented using 8-bit unsigned integers. This choice will significantly reduce the memory and computational costs required by the implementation if the FDI system has many up-down counters. With these choices, the counter value is discrete with a total of 256 possible states. This leaves three parameters to be determined: $\operatorname{Tol}_{1},+U$, and $\mathrm{Tol}_{2}$.

\subsection{Analysis of the Up-Down Counters}

Up-down counters fall within the $\mathrm{G} / \mathrm{D} / 1 / \mathrm{N}$ class of queues in queueing theory. These counters can be modeled using Markov chains (Butto et al. (1991)). The following analysis of the up-down counters is based on Meyn and Tweedie (1993). The counter state is a random variable because the count up/down-count decision depends on the residual. As mentioned above, the counter state is finite dimensional, $c_{k} \in\{0, \ldots, 255\}$. The probability mass function for $c_{k}$ can be represented by the vector $v_{k} \in R^{256}$ :

$$
v_{k}(i):=\operatorname{Pr}\left[c_{k}=i\right]
$$

such that $v_{k}(i)$ represents the probability that the count at time $\mathrm{k}$ is equal to $i$. Here a 0 -based indexing is used for $v_{k}(i)$ for notational simplicity, i.e. $v_{k}(0)$ is the first entry of $v_{k}$. At each point in time the counter state can either count up by $+U$ or count down by -1 , subject to saturation 
limits of the counter. Let $P_{u p, k}$ denote the probability of an up-count at time k, i.e. $P_{u p, k}=\operatorname{Pr}\left[\left|r_{k}\right|>\operatorname{Tol}_{1}\right]$. Thus the probability of a down-count at time $\mathrm{k}$ is $1-P_{u p, k}$. The single step evolution of the counter probabilities can be expressed as:

$$
\begin{aligned}
\operatorname{Pr}\left[c_{k+1}=\min (i+U, 255) \mid c_{k}\right. & =i]=P_{u p, k} \\
\operatorname{Pr}\left[c_{k+1}=\max (i-1,0) \mid c_{k}=i\right] & =1-P_{u p, k}
\end{aligned}
$$

All other transition probabilities from the state $c_{k}=i$ are equal to zero. This evolution can be compactly expressed as $v_{k+1}=M_{k} v_{k}$ where $M \in R^{256 \times 256}$ is given in Equation $(24)$.

$$
M_{k}=\left[\begin{array}{cccccc}
1-P_{u p, k} & 1-P_{u p, k} & 0 & \cdots & \cdots & 0 \\
0 & 0 & 1-P_{u p, k} & 0 & \cdots & \vdots \\
\vdots & 0 & 0 & \ddots & \vdots & \vdots \\
P_{u p, k} & \vdots & 0 & \ddots & 1-P_{u p, k} & \vdots \\
0 & P_{u p, k} & \vdots & \ddots & 0 & 1-P_{u p, k} \\
\vdots & 0 & P_{u p, k} & \ddots & \vdots & 0 \\
0 & \vdots & 0 & \ddots & 0 & \vdots \\
0 & \cdots & \cdots & 0 & P_{u p, k} & P_{u p, k}
\end{array}\right]
$$

The use of this model for analysis relies on the computation of $P_{u p, k}$. This requires a model for the residual. For simplicity, model the residual as $r_{k}=n_{k}+f_{k}$ where $n_{k}$ is zero-mean, Gaussian noise with variance $\sigma_{n}^{2}$ and $f_{k}$ is a fault signal.

The Markov chain defined by Eq. (24) can be used to analyze the false alarm rate. For this case assume $f=0$ so that $P_{u p, k}$ is constant over time. In this case the Markov chain is time-invariant. Denote this constant one-state transition matrix by $M$. This Markov chain is recurrent and it admits a steady-state or stationary distribution $v_{\infty}$. This steady-state distribution is defined as:

$$
v_{\infty}=M v_{\infty}
$$

The probability of being in each counter state does not change when started from the distribution $v_{\infty}$. This distribution is independent from the initial state of the counter. Eq. (25) is simply an eigenvalue problem and can be solved numerically. The eigenvector of the matrix $M$ corresponding to the eigenvalue 1 can be obtained and then normalized by its 1-norm to have sum of probabilities as 1 . The sum of the elements of the $v_{\infty}$ corresponding to the counter states greater than or equal to Tol $_{2}$ yields the false alarm probability.

Fault detection probabilities can be calculated based on the steady-state distribution of the counter states. The problem of interest is that the probability that the counter will exceed $\mathrm{Tol}_{2}$ within a maximum allowable detection time $n$ time-steps. Assume that the probability mass distribution of the counter states before the fault is given by $v_{\infty}$. For this case, assume that a constant bias fault $f$ occurs at time $t=k_{f}+1$. $P_{u p, k_{f}+1}$ again reduces to a constant up-count probability for $k \geq k_{f}+1$. Define $\left.P_{u p, k}\right|_{\text {fault }}=$ $\operatorname{Pr}\left[\left|r_{k}\right|>\operatorname{Tol}_{1} \mid f_{k}=f\right]$ as the up-count probability under faulty operation. A new one-state transition matrix $M_{\text {fault }}$ similar to the one in Eq. (24) can be written with $\left.P_{u p, k}\right|_{\text {fault }}$. The evolution of the counter states over $n$ time- steps after fault can be obtained using $M_{\text {fault }}$. The final distribution is given by $v_{k_{f}+n}=M_{\text {fault }}^{n} v_{\infty}$ based on the constant fault assumption. The fault detection probability $P_{\text {det }}$ is defined as the probability of detecting faults by the end of allowed detection time. $P_{\text {det }}=\operatorname{Pr}\left[c_{k_{f}+n}>\operatorname{Tol}_{2}\right]$ can be calculated as

$$
P_{\text {det }}=\sum_{i=T o l 2}^{U B} v_{k_{f}+n}(i)
$$

where $v_{i}$ is the i-th row of the vector $v$. If the fault $f$ is not constant over the allowed detection time, at each time step a new one-step transition matrix can be calculated. These matrices can be used to propagate $v_{k}$ forward in time until $v_{k_{f}+n}$.

Discrete $+U$ and $T o l_{2}$ parameters to be determined for each fault residual are limited by the size of the counter space, i.e. $0<+U<256$ and $0<$ Tol $_{2}<256$. Tol 1 parameter can be obtained by an equivalent $0<P_{u p, k}<1$ value. $+U, \mathrm{Tol}_{2}, \mathrm{Tol}_{1}$ parameters are determined by a numerical search to maximize detection probability subject to false alarm constraints. This analysis does not hold for the residuals that involve low-pass filters. In this case this solution was used as a baseline and the final values are obtained via iterations on the turbine model. The up-down counter parameters for each fault residual are presented in Table 7 .

Table 7. Up-Down Counter Parameters

\begin{tabular}{cccc} 
Residual No & Tol $_{1}$ & $+U$ & Tol $_{2}$ \\
\hline $1,2,3$ & 1.7753 & 4 & 9 \\
$4,5,6,7,8$ & 0.925 & 2 & 12 \\
$9,10,11,12,13,14$ & 0.55 & 12 & 59 \\
15 & 41.905 & 3 & 4 \\
16 & 0 & 1 & 1100
\end{tabular}

An important benefit of up-down counters is that it can reject large, single event upsets. It is also important for sensors that contain occasional large noise spikes. One such example is aerospace applications where radiation effects can corrupt memory and cause a wildly spurious sensor data. On the other hand up-down counters are more complicated than simple thresholds. Hence it may be preferable to avoid this design if the desired performance can already be achieved by using simple thresholds.

The use of up-down counters differs from straightforward thresholding in two ways. First, the decision to declare a fault involves discrete-time dynamics and is not simply a function of the current value of the residual. Therefore the extra time specified by the detection time constraints can be utilized. Second, up-count and down-count parameters $+U$ and $-D$ introduce a penalty on the residual exceeding the Tol $_{1}$ threshold. Note that simple thresholding is just a special case of an up-down counter. In particular, if $U>(U B-L B)$ and $-D=-U$ then the up-down counter will declare a fault if the residual exceeds $T_{o l}$ for one time step.

The up-down counters can be used as a thresholding algorithm on residuals generated through fault detection filters similar to simple thresholds. However, this introduces a correlated noise signal to the fault residuals. In this case the simple analysis presented above is no longer valid. The analysis of up-down counters with fault detection filters is 
an open area of research topic that is not addressed in this work.

\section{SIMULATION RESULTS}

The performance of the fault detection and isolation system is evaluated via simulations on the benchmark model given by Odgaard et al. (2009). This model is provided as a Simulink model. The designed FDI system is simulated 100 times with different randomly generated noise sequences on each sensor. The wind disturbance given by Odgaard et al. (2009), shown in Figure 5, is used for all simulations. This section summarizes the results of these simulations.

Fig. 5. Wind Disturbance and Turbine Operating Zones
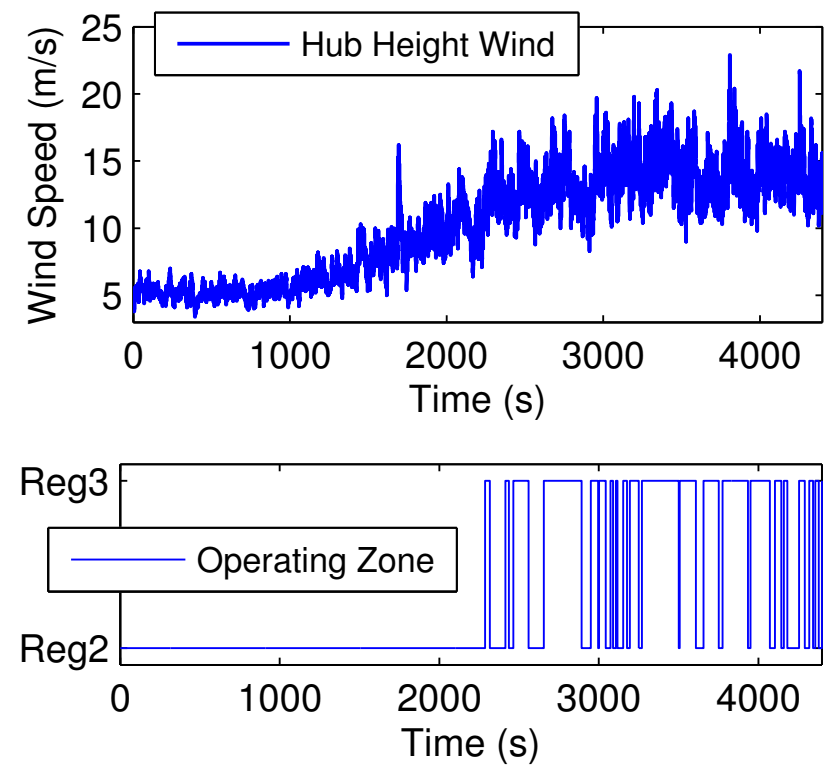

Table 8 lists the average fault detection times, false alarm rates, missed detections, and the average time required for clearing false alarms for each fault. False alarm rate is defined as the number of false alarms per digital processor time step $T_{s}=0.01(\mathrm{~s})$. Fault 5 involves simultaneous faults in two sensors. These sensors are marked in black. Table 8 includes separate detection times for these two sensor. Entries in the table that satisfy the design specifications are marked in green and the failed specifications are marked in red. It is seen in Table 8 that false alarm rate constraints are satisfied for all of the faults. False alarm clearance time requirements are not satisfied for Faults 4 and 5. A pitch sensor fault (Fault 2), faults related to the rotor speed sensors (Faults 4 and 5), and pitch actuator faults (Faults 6 and 7 ) required more time than the specified time limits for detection.

The hydraulic fault in the second pitch actuator (Fault 6 ) is detected approximately 50 seconds after the time of fault. The delay in fault detection is due to the turbine operating in Region 2 during the onset of the fault. The top plot of Figure 6 shows the blade pitch angle commands and positions. The vertical line denotes the onset of Fault 6 . Notice that the blade pitch angles are kept at 0 during the first 50 seconds. Fault 6 remains latent during this period since there is no blade actuator usage. The blade pitch
Table 8. Simulation Results

\begin{tabular}{ccccc} 
F. No & Det. Time & FA Rate & Missed & FA Clear. Time \\
\hline 1 & $3 T_{s}$ & $0.091 / 10^{5}$ & 0 & $2.9 T_{s}$ \\
2 & $819.4 T_{s}$ & $0.046 / 10^{5}$ & 0 & $2.3 T_{s}$ \\
3 & $3 T_{s}$ & $0.046 / 10^{5}$ & 0 & $2.4 T_{s}$ \\
4 & $12.4 T_{s}$ & $0.068 / 10^{5}$ & 0 & $18.8 T_{s}$ \\
5 & $187.4 T_{s} \omega_{r} m 2$ & $0.159 / 10^{5}$ & 0 & $17.5 T_{s}$ \\
6 & $2 T_{s} \omega_{g-m 1}$ & $0 / 10^{5}$ & 0 & $0 T_{s}$ \\
7 & $5050 T_{s}$ & $0.182 / 10^{5}$ & 0 & $3.9 T_{s}$ \\
8 & $1573 T_{s}$ & $0.022 / 10^{5}$ & 0 & $3.2 T_{s}$ \\
9 & $1 T_{s}$ & $0 / 10^{5}$ & 0 & $0 T_{s}$ \\
& $14500 T_{s}$ & 0 & 0 & $0 T_{s}$
\end{tabular}

commands and positions begin to move when the turbine enters Region 3 around time $t=2950 \mathrm{~s}$. It is seen that the fault is detected shortly after the blades begin to move. The fault detection system satisfies the detection time requirements for Fault 6 if the detection time is calculated from the time turbine switches to Region 3 operation.

Fig. 6. Hydraulic Fault in 2nd Pitch Actuator (Fault 6)

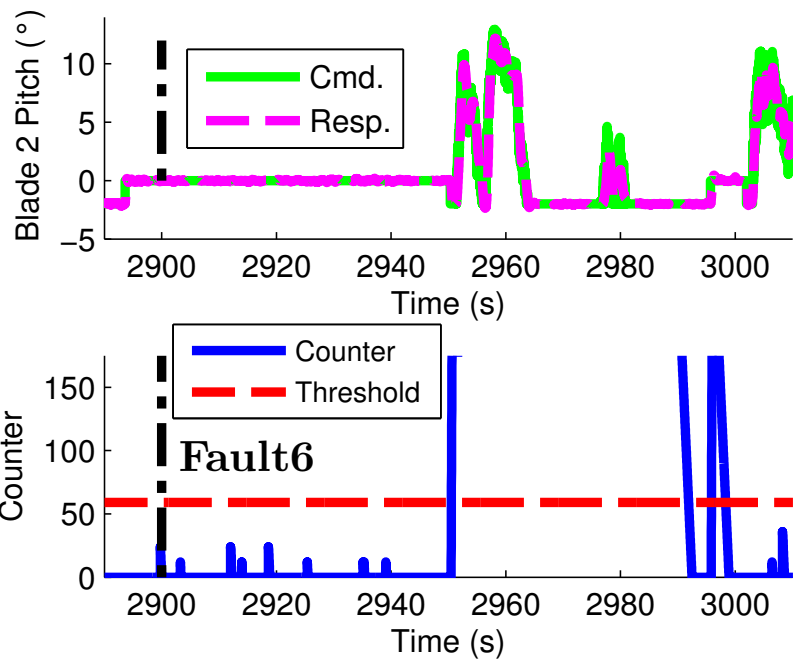

The late detection of Fault 2 is due to the turbine operating conditions for the duration of the fault 2300-2400 (s). The scale factor on the pitch angle can only be detected if the blade pitch angle is large enough to distinguish the fault from the noise. The wind speed is close to the rated wind speed in this time interval. The turbine is operating in Region 3 and generating nonzero pitch commands only during 2300-2320 (s) interval. The fault is detected only for a short duration when the pitch commands reach about 5 degrees around $t=2308(\mathrm{~s})$.

The rotor speed sensor faults (Faults 4 and 5) are hard to detect due to the large noise on the rotor speed sensors. Fault 5 corresponds to a measurement error of about 0.06 $\mathrm{rad} / \mathrm{s}$ whereas the standard deviation of the measurement noise is $0.16 \mathrm{rad} / \mathrm{s}$. The residuals for the rotor speed sensors before and after filtering are given in Figure 7 . Vertical gray lines indicate the onset of the Fault 5. The red lines in the middle plot indicates the up-counting threshold $\left(T o l_{1}\right)$. The bottom plot in Figure 7 shows the counter behavior. Before filtering, the fault occurring at $t=1000 \mathrm{~s}$ is indistinguishable from the sensor noise. The high-order low-pass filtering applied to the fault residual results in a slow detection of faults in these sensors. The failed false 
alarm clearance time constraints are also due to this lowpass filtering since it takes longer for residual to fall below the up-counting tolerance once it is exceeded.

Fig. 7. Rotor Speed Sensor Residual

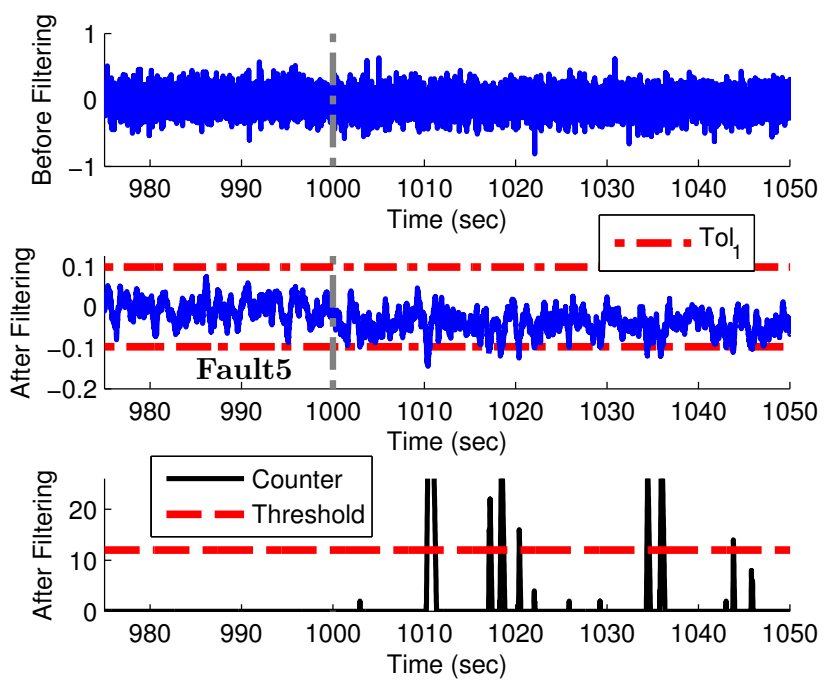

The air-in-oil fault in the third pitch actuator (Fault 7) is detected approximately 16 seconds after the initiation of the fault. This is approximately 10 seconds later than the maximum allowed detection time. This late detection is due to the nature of this actuator fault as well as the wind speed at the time of fault. The fault enters the system gradually and shows its full effect after 30 seconds. Figure 1 shows that the healthy and worst-case faulty actuator models exhibit similar behavior at low frequencies and roll-off at high frequencies. Therefore, pitch actuator faults can only be observed if the pitch commands include large enough components at mid frequency range. At the time of fault initiation pitch commands are relatively small even though the turbine is in Region 3. The fault is detected when the wind speeds up and the actuator system degrades notably.

Fault 9 has been detected successfully by a simple data driven FDI system on the simulation model. However, there are a few potential issues with this simple system when implemented on a fielded turbine. Specifically, this system assumes a constant bias on the wind speed measurements. The detection system needs to be re-trained each time the wind speed sensor is calibrated or there is a change in the measurement bias. Use of more advanced data driven techniques may alleviate these issues.

\section{CONCLUSIONS}

This paper considered use of model-based techniques and up-down counters for wind turbine fault detection of isolation. It is seen that most of the common faults on an industrial wind turbine can be detected with straightforward, easy to implement algorithms. Future work will focus on investigation of more advanced algorithms for drivetrain faults that was found to be challenging for model-based techniques.

\section{REFERENCES}

Butto, M., Cavallero, E., and Tonietti, A. (1991). Effectiveness of the 'leaky bucket' policing mechanism in ATM networks. Selected Areas in Communications, IEEE Journal on, 9(3), 335 -342.

Ding, S.X. (2008). Model-based Fault Diagnosis Techniques: Design Schemes, Algorithms, and Tools. Springer, 1st edition.

Donders, S. (2002). Fault Detection and Identification for Wind Turbine Systems: a closed-loop analysis. Master's thesis, University of Twente.

Emami-Naeini, A., Akhter, M., and Rock, S. (1988). Effect of model uncertainty on failure detection: the threshold selector. Automatic Control, IEEE Transactions on, 33(12), 1106-1115.

Gertler, J.J. (1998). Fault detection and diagnosis in engineering systems. Marcel Dekker, 1st edition.

Isermann, R. (2005). Fault-diagnosis systems: an introduction from fault detection to fault tolerance. Springer, 1st edition.

Johnson, K., Pao, L., Balas, M., and Fingersh, L. (2006). Control of variable-speed wind turbines: standard and adaptive techniques for maximizing energy capture. Control Systems Magazine, IEEE, 26(3), 70 - 81.

Logothetis, D. and Trivedi, K. (1994). Transient analysis of the leaky bucket rate control scheme under poisson and on-off sources. In INFOCOM '94. Networking for Global Communications, 13th Proc. IEEE, volume 2, 490 -497.

Meyn, S.P. and Tweedie, R.L. (1993). Markov Chains and Stochastic Stability. Springer-Verlag, 1st edition.

Odgaard, P.F., Stoustrup, J., and Kinnaert, M. (2009). Fault tolerant control of wind turbines - a benchmark model. In Proceedings of Fault Detection, Supervision and Safety of Technical Processes.

Stoustrup, J., Niemann, H., and la Cour-Harbo, A. (2003). Optimal threshold functions for fault detection and isolation. In American Control Conference, 2003. Proceedings of the 2003, volume 2, 1782-1787.

Szaszi, I., Kulcsar, B., Balas, G., and Bokor, J. (2002). Design of FDI filter for an aircraft control system. In American Control Conference, 2002. Proceedings of the 2002, volume 5, 4232-4237.

Wei, X. and Verhaegen, M. (2008). Fault detection of large scale wind turbine systems: A mixed $H_{\infty} / H_{-}$index observer approach. In Control and Automation, 2008 16th Mediterranean Conference on, 1675-1680. 